\title{
The Structure of 'Phosphatidylglucose'
}

\author{
By N. SHAw \\ Microbiological Chemistry Research Laboratory, Department of Organic Chemistry, University of \\ Newcastle upon Tyne, Newcastle upon Tyne NE1 $7 R U, U . K$. \\ and P. F. SMrTH \\ Department of Microbiology, University of South Dakota, Vermillion, S. Dak. 57069, U.S.A. \\ and H. M. VERHEIJ \\ Biochemisch Laboratorium der Rijksuniversiteit, Utrecht, The Netherlands
}

(Received 4 September 1970)

Smith \& Henrikson (1965) have reported the isolation from Mycoplasma laidlawii strain B of an unusual glucose-containing phospholipid for which a phosphatidylglucose structure (I) was tentatively proposed. Further studies have now established that this structure is untenable and an alternative structure (II), glycerylphosphoryldiglucosyl diglyceride, is now proposed.

The phospholipid was isolated from $M$. laidlawii and purified by the methods previously described (Shaw, Smith \& Koostra, 1968). Hydrolysis with acid (M-hydrochloric acid at $100^{\circ} \mathrm{C}$ for $2 \mathrm{~h}$ ) gave a mixture of fatty acids, glucose and $\alpha \beta$-glycerophosphate. Deacylation of the phospholipid with sodium methoxide yielded a single water-soluble phosphate ester that did not co-chromatograph with a sample of glycerylphosphorylglucose (III), the deacylation product of phosphatidylglucose (I), synthesized from glycidol and glucose (cf. Brown, Hall \& Higson, 1958), or with the isomeric compounds (IV) synthesized from glycidol and $\alpha$ - or $\beta$-glucose 1-phosphate. Treatment of the deacylated phospholipid with sodium borohydride followed by acid hydrolysis still gave glucose and not glucitol, showing that the glucose was bound in the lipid through its reducing group. Hydrolysis with mild acid ( $\mathrm{M}$-hydrochloric acid at $100^{\circ} \mathrm{C}$ for $10 \mathrm{~min}$ ) only partially released the glucose, precluding the presence of a sugar 1-phosphate linkage. The location of the glucose was established from studies of alkaline hydrolysis. Treatment with M-sodium hydroxide at $100^{\circ} \mathrm{C}$ for $1 \mathrm{~h}$ completely hydrolysed the phospholipid to a mixture of fatty acids, diglucosylglycerol and $\alpha \beta$-glycerophosphate. No glucose-containing phosphate esters could be detected. The structure of the diglucosylglycerol was established as $O$ - $\alpha$-D-glucopyranosyl-( $(1 \rightarrow 2)-O-\alpha-\mathrm{D}$ glucopyranosyl-(1 $\rightarrow 1)$-D-glycerol by the methods previously described for the identical glycoside obtained from the glycolipids of $M$. laidlawii
(Shaw et al. 1968). On oxidation the phospholipid consumed $4 \mathrm{~mol}$ of periodate and released $1 \mathrm{~mol}$ of formaldehyde/mol of phosphorus; the deacylated lipid consumed $5 \mathrm{~mol}$ of periodate and released $2 \mathrm{~mol}$ of formaldehyde/mol of phosphorus. All these results are consistent with the proposed glycerylphosphoryldiglucosyl diglyceride structure (II). No evidence is yet available to distinguish between the two possible isomeric structures containing a glycerophosphate residue located on the 6-hydroxyl group of either glucose residue. The phospholipid is completely resistant to hydrolysis by active preparations of phospholipases A, C and D (Verheij, Smith, Bonsen \& van Deenen, 1970), indicating that the glycerophosphate residue is not esterified with fatty acids. Thus the generic term 'phosphatidyl' cannot be applied to this lipid.

Fischer \& Seyferth (1968) have reported the isolation from Streptococcus faecalis and Streptococcus lactis of a glucose-containing phospholipid that yields a diglucosylglycerol and $\alpha \beta$-glycerophosphate on alkaline hydrolysis. Similar degradation products were obtained by Ishizuka \& Yamakawa (1968) on alkaline hydrolysis of a phospholipid from Streptococcus haemolyticus strain D-58, and these authors suggested a diglucosylphosphatidylglycerol structure (V) analogous to the glucosaminylphosphatidylglycerol present in Bacillus megaterium (Op den Kamp, Bonsen \& van Deenen, 1969) and Pseudomonas ovalis (Phizackerley, MacDougall \& Francis, 1966). The results we have obtained are not consistent with a similar structure for the lipid from $M$. laidlawii. Oxidation with periodate of a diglucosylphosphatidylglycerol would not liberate formaldehyde and hydrolysis with alkali should yield detectable amounts of a diglucosylglycerol phosphate, which was not observed. Further studies may show that the glucose-containing phospholipids from streptococci are glycerylphosphoryldiglucosyl diglycerides, thereby emphasizing 


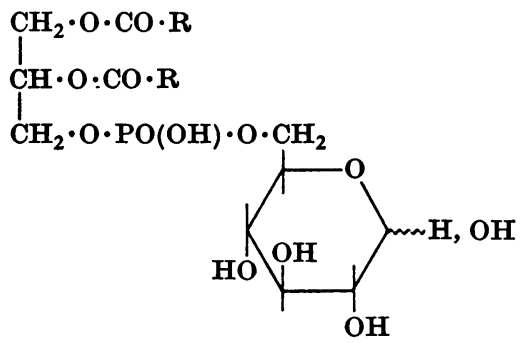

(I)

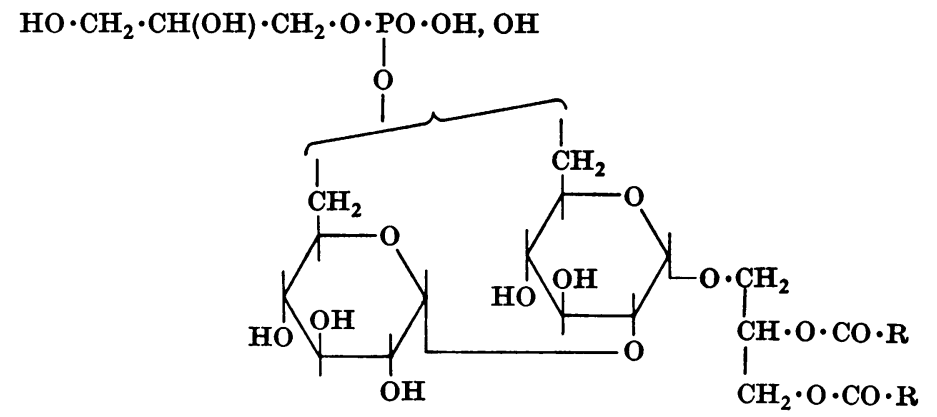

(II)

$\mathrm{HO} \cdot \mathrm{CH}_{2} \cdot \mathrm{CH}(\mathrm{OH}) \cdot \mathrm{CH}_{2} \cdot \mathrm{O} \cdot \mathrm{PO}(\mathrm{OH}) \cdot \mathrm{O} \cdot \mathrm{CH}_{2}$<smiles>OC[C@@]12C[C@H](O)[C@H](O)[C@](O)(O1)[C@@H](O)O2</smiles>

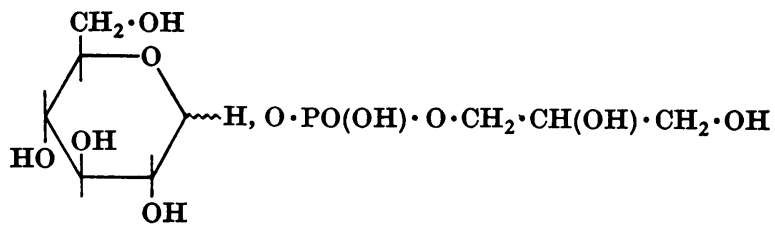

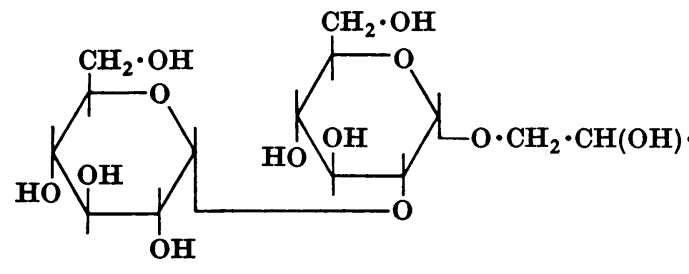

(V)

the similarity in lipid composition previously shown between mycoplasmas and streptococci (Shaw et al. 1968; Plackett \& Shaw, 1967).

While studying the biosynthesis of the glycolipids of Streptococcus faecalis A.T.C.C. 9790 Pieringer (1968) found that three glucose-containing lipids were formed sequentially from UDP-glucose and diglyceride. The first two were identified as monoglucosyl diglyceride and diglucosyl diglyceride, but the third lipid, which was apparently formed from diglucosyl diglyceride, was not identified. However, Pieringer (1968) did report that it was not a triglycosyl diglyceride, but that on deacylation it yielded an anionic water-soluble hydrolysis product. Although he did not identify the nature of the anionic group it seems probable that he was observing the synthesis of a glycerylphosphoryldi- glucosyl diglyceride. The most likely biosynthetic route would be the addition of a glycerophosphate residue to a diglucosyl diglyceride. This route may also be operative in $M$. laidlawii, as the required diglucosyl diglyceride is a major lipid component of this organism (Shaw et al. 1968).

Part of the investigation was carried out under the auspices of the Netherlands Foundation for Chemical Research (S. O.N.), with financial aid from the Netherlands Organization for the Advancement of Pure Research (Z.W.O.) and a grant (AI 04410) from the National Institute of Allergy and Infectious Diseases, U.S. Public Health Service. P.F.S. was recipient of Special Fellowship 1F3AH 38586-01 from the National Institute of Arthritis and Metabolic Diseases.

Brown, D. M., Hall, G. E. \& Higson, H. M. (1958). J. chem. Soc. p. 1360. 
Fischer, W. \& Seyferth, W. (1968). Hoppe-Seyler's Z. Plackett, P. \& Shaw, E. J. (1967). Biochem. J. 104, physiol. Chem. 349, 1662. 616.

Ishizuka, I. \& Yamakawa, T. (1968). J. Biochem., Tokyo, $64,13$.

Shaw, N., Smith, P. F. \& Koostra, W. L. (1968). Biochem. J. 107, 329.

Op den Kamp, J. A. F., Bonsen, P. P. M. \& van Deenen, L. L. M. (1969). Biochim. biophys. Acta, 176, 298.

Smith, P. F. \& Henrikson, C. V. (1965). J. Lipid Res. 6, 106.

Phizackerley, P. J. R., MacDougall, J. C. \& Francis, M. J. O. (1966). Biochem. J. 99, 21 c.

Pieringer, R. A. (1968). J. biol. Chem. 243, 4894.

Verheij, H. M., Smith, P. F., Bonsen, P. P. M. \& van Deenen, L. L. M. (1970). Biochim. biophys. Acta (in the Press). 